University of Nebraska - Lincoln

DigitalCommons@University of Nebraska - Lincoln

P. F. (Paul Frazer) Williams Publications

Electrical \& Computer Engineering, Department

November 1989

\title{
Triggering in trigatron spark gaps: A fundamental study
}

P. F. Williams

University of Nebraska - Lincoln, pfw@moi.unl.edu

F. E. Peterkin

University of Nebraska - Lincoln

Follow this and additional works at: https://digitalcommons.unl.edu/elecengwilliams

Part of the Electrical and Computer Engineering Commons

Williams, P. F. and Peterkin, F. E., "Triggering in trigatron spark gaps: A fundamental study" (1989). P. F. (Paul Frazer) Williams Publications. 23.

https://digitalcommons.unl.edu/elecengwilliams/23

This Article is brought to you for free and open access by the Electrical \& Computer Engineering, Department of at DigitalCommons@University of Nebraska - Lincoln. It has been accepted for inclusion in P. F. (Paul Frazer) Williams Publications by an authorized administrator of DigitalCommons@University of Nebraska - Lincoln. 


\title{
Triggering in trigatron spark gaps: A fundamental study
}

\author{
P.F. Williams and F. E. Peterkin \\ Department of Elecirical Engineering, University of Nebraska-Lincoln, Lincoln, Nebraska 68588-0511
}

\begin{abstract}
(Received 16 March 1989; accepted for publication 20 July 1989)
\end{abstract}
The trigatron has been in widespread use as a demand-triggered, high-voltage switch for more than 40 years. In spite of the popularity and maturity of the technology, there persists an uncertainty over the basic physical mechanism (s) responsible for triggering breakdown in the devices. We present the results of an empirical study of trigatrons that directiy demonstrates that breakdown is initiated by a streamer launched from the trigger pin, independent of the trigger spark. We compare our results with those of previous workers, and discuss the generality of our conclusions.

\section{BAKGROUND}

The trigatron spark gap was first described by Craggs, Haine, and Meek. 'Since that time, the device has found wide application as a demand-triggered, high-voltage switch. In separate applications, trigatrons have been used to switch voltages ranging between several kilovolts and several megavolts. ${ }^{2,3}$ In view of the popularity and maturity of the trigatron, the scientific and engineering literature on the subject is surprisingly inconsistent internally. The problem may be due in part to the large volume of parameter space to be investigated, and in part to the lack of a generally accepted, fundamentally based physical model of the triggering process. In this study we briefy summarize the technical literature and then present empirical results we have obtained recently that clarify the basic physical mechanisms responsible for triggering breakdown in trigatrons.

The trigatron spark gap was invented in the early 1940 s to serve as a switch in high-power modulators for radar. The device was frrst described by Craggs, Haine, and Meek, ${ }^{1}$ and by Wilkinson. ${ }^{4}$ These authors described modulator systems operating in the 10-20-kV, $10-100-A$ range which used trigatrons for switching. Although a number of variations exist, Fig. 1 shows a schematic of a typical trigatron. The gap consists of two main electrodes, with a trigger electrode placed inside a hole in one of them, and insulated from it. In operation, the main gap is charged to a voltage somewhat less than the static, self-break voltage. The switch is closed by triggering breakdown of the main gap through the application of a fast rising trigger pulse to the trigger electrode. After a delay of rypically some tens of nanoseconds, an arc channel forms (usually with the trigger pin as an intermediary) between the main electrodes, and the switch closes.

In these first papers the dependence of the operating characteristics on the voltage polarity configuration was reported, and the exceptional voltage operating range of the device noted. Both blown and sealed devices capable of operation at repetition rates exceeding $1 \mathrm{kHz}$ were reported. Craggs, Haine, and Meek linked the triggering mechanism to "the concentration of the voltage gradient in the region of the trigger wire on the application of the trigger pulse," but attributed the triggering of breakcown to effects of the trigger spark that forms between the trigger wire and the adjacent main gap electrode upon application of the trigger pulse. ${ }^{1}$
Several later reports of studies of trigatron operation have appeared. Of these, most attributed the initiation of breakdown to the action of the discharge between the trigger pin and the adjacent main gap electrode. The most common view ${ }^{1,2,4}$ was that the action of the trigger spark produced a discharge in the main gap, through a Townsend-like mechanism, followed in some cases by a transition to a streamer or Kanal mechanism. Depending on the polarity of the main gap charging voltage, the Townsend-like discharge was thought to be initiated by electrons produced by photoemission from the distant cathode, photoionization of the gas in the gap, or drift from the plasma of the trigger spark. These electrons would create others through electron impact ionization, and eventually cause either the formation of a transient glow discharge between the main gap electrodes or a streamer. Further heating would then occur to form the arc and close the switch. Sletten and Lewis ${ }^{5}$ and Lampe ${ }^{6}$ proposed that another factor in the breakdown of the nominally undervolted main gap resulted from the expansion of hot gases from the trigger spark into the main gap region. Because at a fixed pressure the molecular (or atomic) number density of a gas decreases with temperature, $E / N$ and therefore the Townsend ionization coefficient $\alpha$ would be higher in the hot gas. Saxe proposed a similar mechanism, but inroked the shock wave propagating outward from the trigger spark to produce the entanced $E / N$.

Craggs $e t a l^{2}$ suggested that triggering might be due to the field distortion around the trigger pin tip. Subsequent authors generally discounted this mechanism, however, on the basis that breakdown of the main gap in many cases occurred after the breakdown of the trigger gap. They rea-

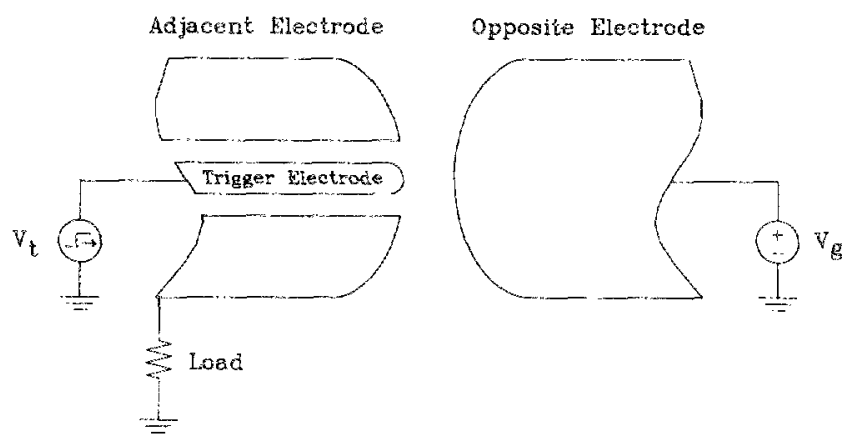

FIG. 1. Schemakic drawing of a Yypical trigatron spark gap. 
soned that since the voltage on the trigger pin collapses upon breakdown of the trigger gap, the breakdown process in the main gap must be arrested at the same time in it is initiated by field distortion. In a remarkably insightful paper, Shkuropat ${ }^{8}$ noted that the clear depencence of trigatron behavior on the relative polarities of the trigger and main gap charging voltages is difficult to explain with mechanisms of this type. Triggering was almost universally observed to be better with the heteropolar configuration than with the homopolar. Since the voltage on the trigger pin collapses upon breakdown of the trigger gap, this difference is difficult to explain in terms of a model in which all the action occurs after this collapse. On the basis of experiments conducted with a $\approx 100-\mathrm{kV}$ trigatron, he concluded that, at least for charging voltages resulting in rapid triggering, the initiating events occur before the collapse of the trigger gap voltage, and suggested that a streamer initiated directly from the enhanced field around the trigger pin tip was involved. With this model it is only necessary that the trigger gap not breakdown until after the streamer has been initiated and, perhaps, traversed some portion of the gap. Further experiments reported in a later paper ${ }^{9}$ supported this model, and explored the transition from streamer to arc channel.

In this model, the enhanced field near the trigger pin tip launches a streamer that propagates across the gap, bridging it with a low-conductivity channel. This channel then heats to form the arc which closes the switch. Propagation of the streamer tip depends on the presence of an enhanced field ahead of it. This field is determined by a number of factors, including the algebraic difference between the potential of the tip and that of the opposite main gap electrode. Since the tip is connected to the trigger pin through the streamer channel, before the trigger potential collapses this difference is substantially larger for a heteropolar than for a homopolar configuration. This fact then explains quaitatively the observed dependence of trigatron characteristics on polarity configuration. Further, the model predicts that increasing trigger voltage will improve triggering only up to that point where the trigger gap breaks down before the streamer has traversed the gap. Thus the design of an optimum triggering system involves balancing the requirements of maximizing the field enhancement near the trigger pin tip, with delaying the breakdown of the trigger gap at least until a streamer can be formed and propagate across the main gap. Shkuropat ${ }^{9}$ reported behavior consistent with this prediction.

In a series of papers, Yoshida and Sugita ${ }^{10,11}$ reported the results of experimental and numerical modeling studies of trigatrons. They proposed that two distinct modes of breakdown exist, which they refer to as longitudinal trigger and side trigger. The longitudinal trigger mode is similar to that discussed by Shkuropat, ${ }^{8,9}$ and the side trigger mode is similar to the mode proposed in the earlier papers. ${ }^{1,2,6}$ No explanation was provided as to why the longitudinal mode is observed in some cases and the side mode in others, and they reported that their gap operated only in the side trigger mode. This latter conclusion was based, however, mainiy on their observation that breakdown occurred in the trigger gap before the main gap. This observation does not exclude the streamer mechanism. Further, Yoshida and Sugita present- ed time-resolved shutter photographs of the emission from the gap during triggering. These photos show diffuse regions of luminosity propagating from the trigger to the opposite main gap electrode at a speed of $\approx 10^{8} \mathrm{~cm} / \mathrm{s}$, too fast to be due to electron drift. Although Yoshida and Sugita interpret these data in terms of the side trigger mode of operation, they seem actually to be more supportive of the longitudinal mode instead.

El'chaninov et al..$^{12,13}$ and Emel'yanov et al. ${ }^{14}$ have published results of experimental studies of high-voltage ( 400 $\mathrm{kV}$ to $1.9 \mathrm{MV}$ ) trigatron operation. Through the use of up to eight trigger pins in the same gap, these authors report simultaneous closure through multiple are channels with current closure with current sharing between the arcs. The design of their gaps was based on the streamer-initiated breakdown model, and they presented experimental results supporting the validity of it. Particularly convincing are measurements of closure delay versus trigger voltage that show that the delay first decreases, then increases with increasing trigger voltage, similar to the results of Shkuropat. ${ }^{8,9}$ In later work, Kremnev, Novakovskii, and Potalitsyn $^{15}$ studied arc channel formation in a high-voltage trigatron with nanosecond time resolution, and presented shadowgraphs of the developing arc channel in nitrogen for charging voltages between about $80 \%$ and $200 \%$ of static self-break.

Wootton ${ }^{16}$ studied a irigatron spark gap operating near the minimum triggerable main gap voltage, and concluded that main gap breakdown was initiated by streamers. He presented photographic evidence showing that the main and trigger gap arcs connected to different points on the surface of the trigger pin, and developed a triggering map that clearly showed the deleterious effect of too high a trigger voltage, causing early breakdown of the trigger gap.

Martin has developed a phenomenological description of breakdown, which he has applied to a number of devices, including trigatrons. ${ }^{17}$ The description is based on a model in which breakdown is initiated by a streamer (which he calls a fast discharge) crossing the gap, and completed by a heating phase in which the weakly conducting channel left by the streamer is transformed into an arc. Working with Martin, Wells obtained photographs that show the filamentary nature of the initiatory processes in a trigatron. ${ }^{18}$

We report here results of an extensive experimental program investigating the basic physical mechanisms responsible for initiating breakdown in trigatrons. Besicies the usual current and voltage diagnosties, this program made use of high-speed, high-sensitivity streak and shutter photography to study the triggering mechanism over a wide range of parameter space. Brief reports of this work at various stages have been presented by Wages et $a l^{19}$ and Peterkin and Williams. ${ }^{20,21}$ As a result of this program, a much clearer picture of the physical mechanisms responsible for initiating breakdown in trigatrons has emerged. This picture is discussed in detail in the next section.

\section{EXPERIMENTAL APPARATUS}

Figure 2 shows a schematic diagram of the experimental setup. The trigatron spark gap was contained inside a spe- 


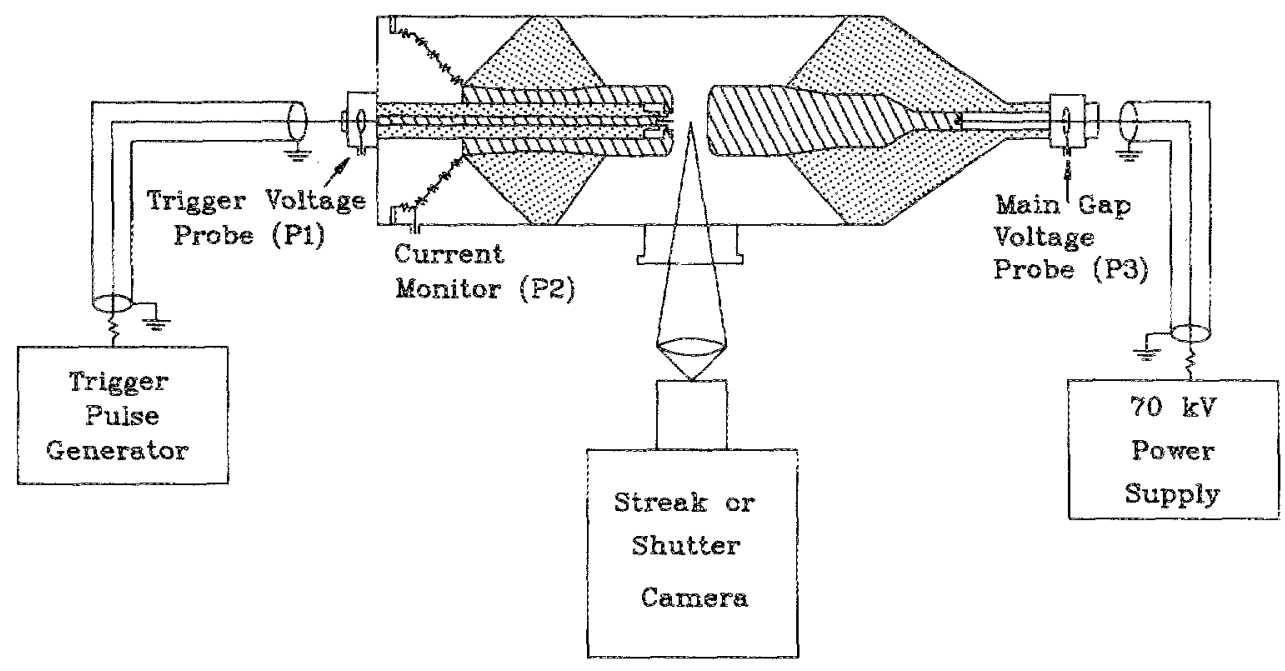

FIG. 2. Schematic diagram of the experimental setup used for the work discussed in this paper.

cially designed cell which could be evacuated and then backfilled to any pressure up to about $2 \mathrm{~atm}$, absolute. The inside diameter of the cell was $14.22 \mathrm{~cm}$, and the outside diameter of the electrodes was $6.06 \mathrm{~cm}$. The cell was designed to operate with charging voitages up to $70 \mathrm{kV}$. Care was taken to ensure that the cell appeared electrically as a constant-impedance, 50- $\Omega$ coaxial transmission line. A 4 -in., fused quartz window provided optical access to the gap. The main gap electrodes were construeted of brass machined to a Chang constant-field profile using a numerically controlled lathe. ${ }^{22}$ With a $2.5 \mathrm{~cm}$ gap separation, the static self-breakdown voltage $V_{S B}$ for 700 - Torr $N_{2}$ was $63 \mathrm{kV}$. Lexan supports, shaped as indicated in the figure to maintain the $50-\Omega$ transmission line impedance, insulated the electrodes from the cell wall. The gap was charged through a length of $R G$ $218 / \mathrm{U}$ coaxial cable. A short, $24 \mathrm{~ns}$ length of cable was used for high-sensitivity photographic experiments looking at the initial breakdown processes to avoid swamping the camera with the intense emission from the arc. A longer, 240 ns length was used in experiments investigating the time to breakdown and other features of the arc formation phase.

The main gap electrode containing the trigger pin was comected electrically to ground through a specially designed $50-\Omega$ load. This load resistor consisted of 18 seriesconnected coaxial rings, each containing thirty-six 100-n, 2W carbon composition resistors, mounted coaxially inside a cylindrical extension of the spark gap housing. Dorningos has shown that these resistors remain linear for low duty cycle voltage pulses of up to $2 \mathrm{kV}$. ${ }^{23}$ The load resistor should therefore remain linear up to the $70-\mathrm{kV}$ charging voltage design limit. The diameter of each ring was chosen so that the characteristic impedance of the transmission line at the point would just equal the remaining resistance to ground. For monitoring the gap current, a $0.5-\Omega$ current viewing resistor ring (labeled $\mathrm{P} 2$ ) consisting of 36 parallel-connected $18-\Omega, \frac{1}{8}-W$ carbon composition resistors was placed in series with the load resistor at the grounded end. To minimize inductive overshoot in the diagnostic, the ring fit snugly inside the cylindrical outer conductor, and was insulated from it with a thin layer of tefton tape. We estimate the $L / R$ response time of the current monitor to be of the order of 100 ps, and the overall rise time to be limited by our oscilloscope (1-2 ns). We saw no evidence of inductive overshoot, but current measurements were complicated by capacitive coupling between the load resistor and the conductor connecting to the trigger pin that ran down the axis of the resistor.

The trigger puise was provided by a laser-triggered spark gap connected to a length of charged RG-8/U coaxial cable. When fired into a matched load, the laser-triggered gap produced pulses with a rise time of $4-5 \mathrm{~ns}$, with jitter as measured from the laser pulse of about \pm 1 ns. The charged cable was energized with a regulated power supply. Pulses of magnitude between about 5 and $25 \mathrm{kV}$ conld be delivered to the trigger pin.

The trigger generator was connected to the trigger pin through a short $(\approx 30 \mathrm{~cm})$ length of RG-8/U cable that was connected to a $\frac{1}{4}-\mathrm{in}$. steel drill rod coaxial with the cell axis, and insulated with a l-in.-diam Lexan sleeve. The diameter of the hole in the main gap electrode in which the trigger pin was placed was $1 \mathrm{~cm}$. In order that a wide range of configurations could be studied, the end of the drill rod was threaded so that various trigger pins could be used and an interchangeable Macor sleeve was used to insulate the trigger pin from the adjacent electrode. Trigger pins with both a rounded and a squared-off tip, with diameters ranging from 0.08 to $0.48 \mathrm{crn}$, as well as a ring-shaped electrode were studied. The position of the pin tip was also varied from lying below the surface plane of the main gap electrode to fush with it. A configuration in which the insulator was fush with the main gap electrode surface was also studied.

The electrical diagnostics consisted of two capacitively coupled voltage probes and the current-viewing resistor discussed above. The voltage probes monitored the trigger voltage at the point where the coaxial cable from the trigger generator entered the cell, and the voltage on the charged main gap electrode where the main charging cable entered the cell. Both probes consisted of a thin metal ring of the same inner diameter as the shield of the coaxial cable, and insulated from ground by plastic spacers. The insulated center conductor of the coaxial cable passed through the ring. The voltage on the ring was monitored using a Tektronix \$6009-100 probe for the trigger voltage (iabeled $\mathrm{PI}$ ), and 
a Tektronix $\mathrm{P} 6063 \mathrm{~B} \div 10$ probe for the main gap charging voltage (labeled $\mathrm{P} 3$ ). The division ratio of a voltage diagnostic is determined by the ratio of the capacitance between the ring and the inner conductor to the capacitance between the ring and ground (including the capacitance of the Tektronix probe), and by the division ratio of the Tektronix probe. For $P 1$, the overall division ratio was $3500 \pm 200$, and the rise time when used with a Tektronix 7834 storage oscilloscope was about 2 ns. For P3, the division ratio was $1650 \pm 100$, with a rise time of about $1 \mathrm{~ns}$. Since voltage is applied to the gap through a $50-\Omega$ coaxial cable, the voltage changes measured at $\mathrm{P} 3$ are also an accurate measure of the gap current, at least for times less than the roundtrip transit time of the cable.

High-speed, high-sensitivity streak and shutter cameras were used to obtain information about the early stages of triggered breakdown in the trigatron cell. Both camera systems have suficient gain that single photoelectrons emitted from the photocathode can be detected. The input optical systems for the streak and shutter cameras were about $f / 4$ and $f / 6$, respectively. The photocathodes in both systems have about $10 \%$ quantum efficiency in the visible and nearultraviolet. The streak camera system consisted of the Hamamatsu C979 streak camera with type N895-01 photocathode, (spectral range of 200-700 nm); an M1284 slow plug-in unit giving streak times between 10 and $500 \mathrm{~ns}_{\text {; }} \mathrm{Cl} 1795$ gate generator to protect the camera from the intense emission from the fully developed arc; and C1000-18 SIT vidicon camera and C2280 temporalanalyzer for image acquisition, digitization, and analysis. The acquired image was viewed on a raster-scan video monitor, and could be permanently stored by photographing the monitor with a Polaroid or 35$\mathrm{mm}$ camera. The emission from the trigatron gap was imaged onto the entrance slit of the streak camera with a 50$\mathrm{mm}, f / 1.4$ photographic lens. The entrance slit for all data reported here was $100 \mu \mathrm{m}$ wide. With the input optical system we used, the streak camera viewed an approximately 1.2-mm-wide region of the trigatron gap, centered on the axis.

The shutter camera was locally constructed. It consisted of an TTT F-4144 dual microchannel plate image intensifier tube specially modifed for fast gating, gate generator, and associated power supplies. The intensifier tube has an \$-25 photocathode with a similar spectral response as the streak camera tube. The gate generator was a locally designed and constructed avalanche transistor circuit capable of delivering pulses as short as about $5 \mathrm{~ns}$ to the intensifier tube. The camera was designed so that the Kamamatsu image acquisition electronics (consisting of the C1000-18 SIT vidicon camera and the C2280 temporalanalyzer) could be used to acquire images from the shutter camera in a manner similar to that used with streak pictures.

To synchronize the electrical and optical diagnostics, a small portion of the laser beam used to fire the laser-triggered spark gap was picked off and directed onto the trigatron cell window where it could be viewed by the camera system. Another small portion of the laser beam was directed toward a fast photodiode (HP 5082-4220) which provided an electrical signal. By carefully measuring electrical and optical path lengths, the timing between the optical and electrical events was accurately known, allowing synchronization of the optical and electrical diagnostics to within about \pm 1 ns.

\section{PHYSICAE MECHANISM OF BREAKDOWN}

This work has shown that the following sequence of events is responsible for triggering breakciown in trigatron spark gaps in the region of parameter space (charging voltage near self-break, trigger voltage $20 \%-50 \%$ of charging voltage, and roughly atmespheric pressure) in which they are normally operated. Upon arrival of the trigger pulse, streamers form after a short delay and propagate across the gap. One or more (usually several) streamer channels then connect the trigger pin to the opposite main gap electrode through a resistance of the order of $10 \mathrm{k} \Omega$, and the switch is still effectively open. The applied field causes the ionization density in these streamer channels to rise, decreasing the resistance. In almost all cases the density in one of these channels grows faster than the others and dominates, eventually forming an arc. Concurrently, the gap between the trigger pin and the adjacent main gap electrode also undergoes a streamer/channel-heating breakdown process. The detailed sequence of events beyond this point is complex, depending on the relative timing of these two breakdown processes, the source resistance and pulse length of the trigger generator, and the main gap charging voltage. In most cases the final result is two thermalized arcs connecting the trigger pin to the opposite main gap electrode and the adjacent electrode, but other final configurations are probably possible.

The main gap breakdown process can be divided into three phases: streamer initiation, streamer propagation across the gap, and subsequent heating to form the arc channel. We will discuss each of the phases separately. The discussion will center around operation in the normal region of parameter space for these gaps as defined above, but some data for operation outside this region will also be discussed. In particular, in the last section, we will discuss triggered breakdown with charging voltage well below self-break $\left(25 \%-70 \%\right.$ of $\left.V_{\mathrm{SB}}\right)$.

\section{A. Streamer initiation}

In order to form a streamer, a small region of plasma must be created with an ionization density high enough to shield itself at least partially from the external field. A general rule of thumb is that a plasma consisting of about $10^{8}$ free electrons and positive ions is required. ${ }^{24}$ There is little direct evidence showing how this plasma is formed, but the most likely source is single, seed electrons that rapidly avalanche in the high electric field near the trigger pin tip. For a $2-\mathrm{mm}$ trigger gap and $20-\mathrm{kV}$ trigger voltage, and neglecting field enhancements due to geometry and main gap feld, a single electron would avalanche into $10^{8}$ electrons in about 1 ns in atmospheric pressure $\mathrm{N}_{2}$, and the avalanche length would be about $0.3 \mathrm{~mm}$. Including geometric and applied field enhancement effects would shorten both the formative time and the avalanche length. 
There is also little evidence about the source of the seed electrons required to initiate the avalanche. One possible source is the raturally occurring free electrons in the highfield region around the trigger pin tip. We estimate the number of free electrons in this region as

$$
N=2 \pi r^{2} n_{0} d r
$$

where $r$ is the radius of the (assumed hemispherical) pin tip, $d r$ is a measure of the length of the enhanced field region surrounding the tip, and $n_{0}$ is the naturally occurring backgrounc density of free electrons. Morgan estimates the freeelectron density in atmospheric air to be $500 \mathrm{~cm}^{-3} \cdot{ }^{25}$ Assuming this ionization to be uniformly distributed, and taking $d r=2 \mathrm{~mm}$, we estimate that $N$ ranges from a few for a small diameter trigger pin tip $(r \approx 0.5 \mathrm{~mm})$ to several hundred for a large pin $(r \approx 0.5 \mathrm{~cm})$. The assumption of uniform distribution is questionable, however, because the most likely sources of the ionization are tracks left by cosmic rays and other naturally occurring ionizing particles.

This estimate suggests that for trigger pin diameters normally used in trigatrons there should be a sufficient number of natural electrons to initiate reliably the requisite streamer, but for small diameter pins, statistical fuctuations in the density of these electrons may become important. Our experiments show that several streamers are typically initiated near the trigger pin tip during the triggering process in trigatrons, and that the number of these streamers increases with the size of the trigger pin. These results are consistent with the assumption discussed above, but they are not conclusive. Photoionization from either surface or voiume corona occurring within the trigger may well also be an important source of seed electrons. The technical literature is divided on this point, however. Some workers report improved performance with trigger pins designed to enhance corona formation, ${ }^{4,9}$ whereas others report no effect. ${ }^{5}$

\section{Streamer propagation}

This stage of the breakdown process is the best documented. Figure 3 shows a time sequence of two-dimensional shatter photos of the propagation of cathode-directed streamers in the trigatron gap taken with $V_{\mathrm{g}}$ near $V_{\mathrm{SB}}$. The shutter was open for about $5 \mathrm{~ns}$. Since the rransit time for the streamers was also of the order of $5 \mathrm{~ns}$, there is considerable motional blurring of the images. In Fig. $3(b)$, for example, it appears that the luminosity is fairly uniform along the streamer channel, whereas streak photos show that the region just behind the tip is generally more luminous than the rest of the streamer body. Also shown are the positions of the main gap electrodes and the trigger pin, drawn to scale. Unfortunately, for each breakdown event only one such picture could be obtained with our apparatus, so these photos are each from a different shot. There was a 5-10-ns jitter in the time of appearance of the first streamer relative to the timing of the trigger voltage pulse. The times listed in the figure are times since the leading streamer was initiated as estimated from streak photographs of streamers under the same conditions.

A number of interesting features are evident. Several streamers are launched from the vicinity of the trigger pin

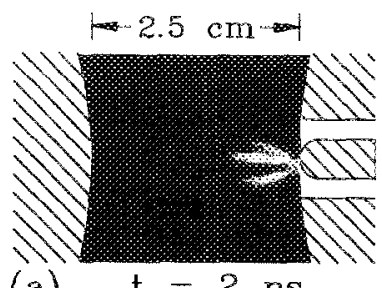

(a) $\mathrm{t}=2 \mathrm{~ns}$

(b)

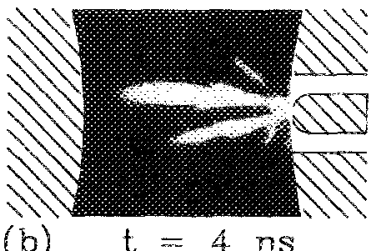

$\mathrm{t}=4 \mathrm{~ns}$

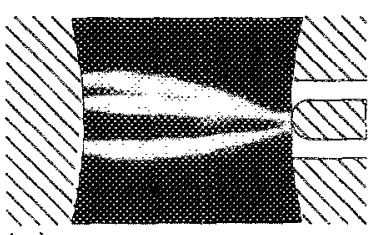

(c)

$t=10 \mathrm{~ns}$

(d)

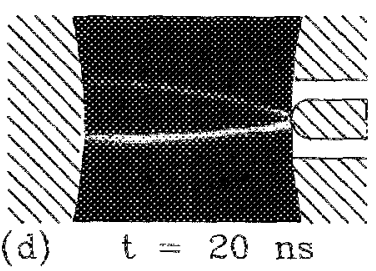

FIG. 3. Sequence of shuter photos showing the time development of cathode-directed streamers in the main gap. The streamers are pictured at various times after initiation. Due to the increasing intensity of the channels, the image intensifier gain was lower for $(c)$ and (d). Conditions were: positive trigger, negative main gap $(+-)$ polarity, $V_{t}=10 \mathrm{kV}, V_{g}=-60$ $\mathrm{kV}, \mathrm{N}_{2}$ at 700 Torr, $2.5 \mathrm{~cm}$ gap separation, and 4.76-mm-diam trigger pin flush with the main electrode.

tip. These streamers have diameters of $1-2 \mathrm{~mm}$, and travel across the gap roughly following field lines. After contacting the opposite electrode the luminosity increases. One channel typically increases more rapidly than the rest and eventually dominates. The camera sensitivity is lower in the last two photos than in the others, and is lower in the 20 ms photo than in the 10-ns photo. We obtained shutter photos similar to those in Fig. 3 for a number of pressures between 250 and 900 Torr in $N_{2}$. Except for a weak dependence of velocity on pressure, the characteristics of the streamers appeared similar at all pressures. Specifically, little variation in streamer diameter was seen over this range.

Figure 4 shows a shutter photograph of an anode-directed streamer, taken under conditions similar to those for Fig. 3. For main gap charging voltage near $V_{S B}$ the main difference between the photos we obtained of anode-directed and cathode-directed streamers is the more pinnate appearance of the anode-directed streamers. The propagation speed was

\section{$1-2.5 \mathrm{~cm}-1$}

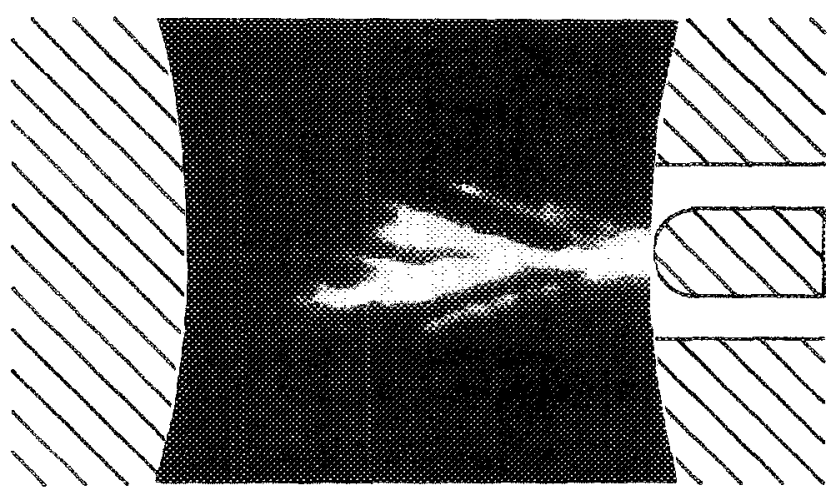

FIG. 4. Shutter photograph of anode-directed streamer ins the negative trigger, positive main gap $(-+)$ polarity, $V_{t}=-10 \mathrm{kV}$, and $V_{g}=60 \mathrm{kV}$. Other conditions were the same as in Fig. 3. 
a intle slower than that of cathode-directed streamers. As discussed in a later section, however, the behavior of the anode-directed streamers changed markedly with decreasing main gap voltage.

Streak photography provides information complementary to that from shutter photography. Figure 5 shows a streak photograph of a streamer crossing the main gap in our trigatron, along with the gap current measured on the same shot. The current was measured by monitoring the voltage at P3 and noting that for times less than a roundtrip transit time of the $50-\Omega$ coaxial charging cable, the voltage changes and current are related by Ohm's law. The operating conditions were similar to those in Fig. 3. As the streamer passes a fixed point in the gap, the detected emission intensity from that point increases abruptly, then decreases with a time constant of roughly 2 ns. The abrupt increase shows that the tip of the streamer is sharp and spatially well defined, and the decrease after the passage of the streamer tip shows that the electric field is significantly less (causing a smaller electron impact excitation rate) inside the streamer than at the tip. The observed 2-ns decay rate is somewhat faster than the 40ns low-pressure lifetime of the $v^{\prime}=0$ and $v^{\prime}=1$ levels of the $C\left({ }^{3} \Pi_{u}\right)$ state of $\mathrm{N}_{2},{ }^{26}$ but is probably consistent considering that the gap was operated at near atmospheric pressure. The speed of the streamer varies from about $2 \times 10^{8} \mathrm{~cm} / \mathrm{s}$ at initiation to more than $2 \times 10^{\circ} \mathrm{cm} / \mathrm{s}$. This behavior and these speeds are consistent with numerical modeling predictions. ${ }^{27,28}$

The time scales on the streak photo and the current trace in Fig. 5 are synchronized to within \pm 1 ns. Starting within a few nanoseconds of the time the streamer appears at the trigger pin tip on the streamer photo, the gap current starts to rise. This current is the result of the motion of free electrons in the streamer tip, ahead of the streamer (produced by photoionization or photoemission), and inside the streamer body. The plasma of the streamer tends to shield the streamer interior from the external field, but is only partially successful because of the rapidly changing conditions produced by the propagating streamer tip. The gap current rises pri-

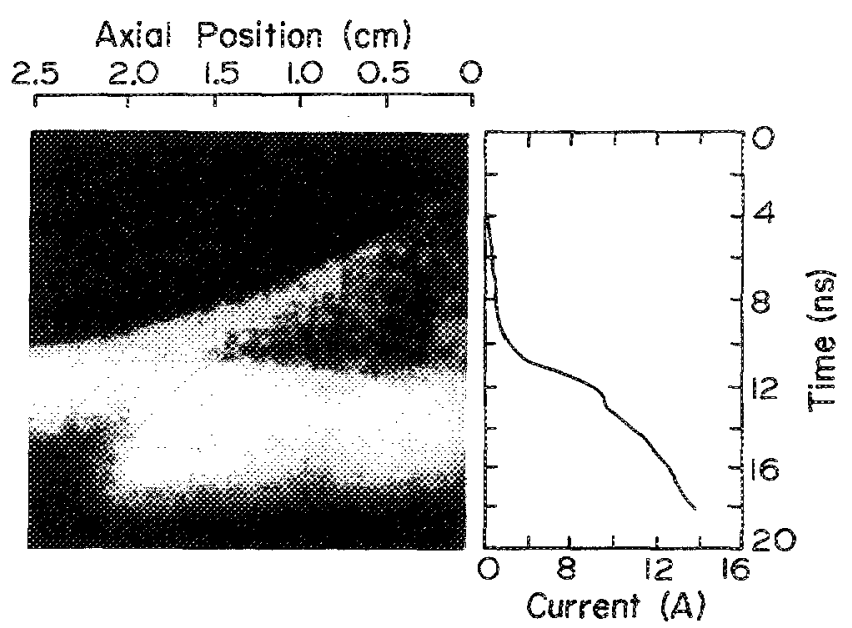

FIG. 5. Synchronized streak picture and main gap current trace, obtained under the same conditions as Fig. 3. Synchronization is accurate to within \pm 1 ns. marily because the number of free electrons inside the streamer body increases as the streamer channel lengthens. As the streamer nears the distant electrode, shielding of the interior becomes increasingly difficult because the external circuit maintains a constant potential drop between the trig. ger and main gap electrodes. Some of the current increase may, therefore, also be due to a decrease in shielding efficiency.

In most cases the gap current jumped simultaneously ( $\pm 1 \mathrm{~ns})$ with the streamer arriving at the opposite main gap electrode. When the streamer contacts this electrode, the requirement of constant potential drop is inconsistent with significant shielding of the main streamer body, and the field inside the streamer must rise. ${ }^{29}$ This effect is seen in the electrical diagnostic as this current jump, and in the optical diagnostic as a sudden increase in luminosity. Using the gap current just after the jump and neglecting any voltage drop across the electrode-plasma interfaces, we estimate the resistance of the streamer channel at this time to be somewhat larger than $6 \mathrm{k} \Omega$, and the average free-electron density in the streamer chamnel to lie in the range $10^{14}-10^{15} \mathrm{~cm}^{-3}$, in good agreement with theoretical expectation. ${ }^{27,28}$

Simultaneously with the process described above, the gap between the trigger pin and the adjacent main gap electrode is also undergoing a streamer-initiated breakdown process. We obtained two-dimensional shutter photographs of the early events in the trigger pin well by using a right angle prism to look directly into it. In these experiments the fill gas was ambient air and the main gap was uncharged. In photos taken within 10-20 ns of the arrival time of the trigger pulse we typically saw about ten streamerlike channels in a radial pattern. Subsequent photos showed one channel brightening more than the others, and eventually dominating and forming an arc channel, as in the main gap.

The progress of the breakdown of the trigger gap could be monitored with the gap current monitor (P2 in Fig. 2), and with optical diagnostics. For charging voltage close to $V_{\mathrm{SB}}$, breakdown of the trigger gap clearly occurred well after streamers had been initiated and traversed the main gap. For lower charging voltages, as discussed in Sec. III D, the streamers propagated more slowly, and the trigger gap spark formed while the main gap streamers were still in transit.

We have obtained streak photographs of streamers initiating triggered breakdown in our trigatron over a wide range of conditions. The dependence of streamer velocity on environmental parameters such as applied voltage or pressure is a quantity of obvious interest. Since there are a range of velocities associated with each streamer, we chose to tabulate the time required for the streamer to propagate across the gap as a single-number measure of velocity. Figure 6 shows (as the open symbols) such a tabulation as a function of main gap voltage, $V_{g}$. Data are presented for a set of four trigger voltages ranging from 10 to $25 \mathrm{kV}$, and for the two heteropolar charging configurations. The polarity notation used in the figure and throughout this study consists of two sequential signs. The first sign gives the polarity of the trigger voltage referenced to the grounded main gap electrode, and the second gives the polarity of the opposite, charged, main gap electrode with the same reference. 

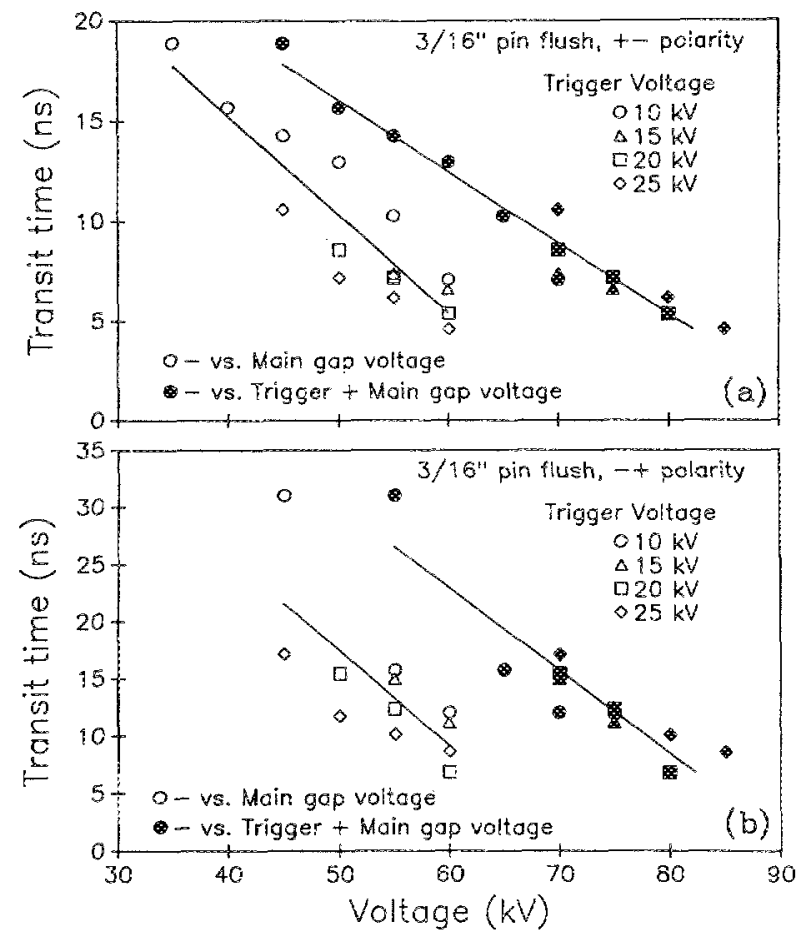

FIG. 6. Plot of stremer transit time vs main gap voltage and vs main gap plus trigger voltage, for (a) positive trigger, negative main gap $(+-)$ and (b) negative trigger, positive main gap $(-+)$ polarity. Conditions were otherwise the same as in Fig. 3.

Since the streamer velocity is a function of (among other things) the electric field ahead of it, and since the streamer consists of a weakly conductive channel connected to the trigger pin, one might expect that the streamer velocity would be more accurately a function of the voltage difference between the trigger pin tip and the distant main gap electrode, $V_{g}-V_{t}$, than the difference between the two main gap electrodes, $V_{g}$. This point was first made by Martin. ${ }^{17}$ Also shown in Fig. 6 (as filled-in symbols) are the same transit time data plotted vs $\left|V_{g}-V_{i}\right|$. The scatter is clearly reduced in the second set of points. The solid lines are least-squares fits to the data. The correlation coefficients for the $V_{g}-V_{t}$ sets were 0.98 and 0.92 for the +- and -+ polarities, respectively, whereas they were 0.91 and 0.75 for the $V_{8}$ set. We therefore conclude that a more meaningful representation of the streamer data is provided by tabulating it as a function of $V_{g}-V_{t}$ rather than just $V_{g}$.

For charging voltages near $V_{\mathrm{SB}}$ we have tabulated the gap transit times as a function of trigger pin diameter, and find that within experimental scatter the dependence is weak. Figure 7 shows a tabulation of streamer transit time as a function of flll gas pressure for pressures between 250 and 900 Torr for charging voltages kept a fxed fraction of $V_{S B}$. At the higher pressures there is a weak dependence on pressure that becomes more pronounced as the pressure decreases. These data were, however, taken with a fixed trigger voltage. It probably would have been more meaningful to reduce the trigger voltage synchronously with the pressure reduction in order to keep the trigger voltage a fixed percentage of the charging voltage. Doing so would result in a stronger dependence of streamer transit time on pressure than we found.

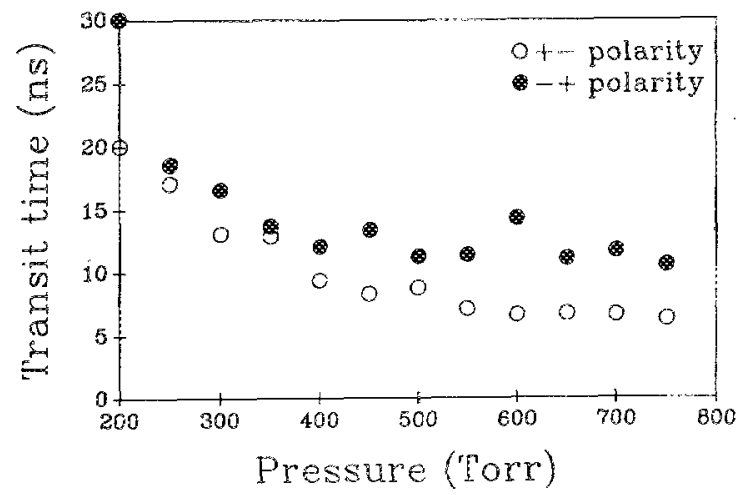

FIG. 7. Plot of streamer transit time for varied pressure at fixed trigger voltage, $\left|V_{t}\right|=10 \mathrm{kV}$, and for both polarity configurations. The main gap charging voltage $V_{g}$ was maintained at $99 \%$ of $V_{5 B}$ and conditions were otherwise the same as in Fig. 3.

Finally, we found that in all cases for which conditions were otherwise equivalent, cathode-directed streamers (observed in the +- polarity configuration) traversed the gap faster than anode-directed streamers (observed in the -+ configuration). This observation is somewhat surprising since the cathode-directed streamer travels in a direction opposed to the force exerted on the electrons by the electric field, and since computer simulations of streamer propagation for simplifed conditions generally predict that the cathode-directed streamers are slower.

\section{Channel heating and arc formation}

Figure 8 shows an oscillogram of the main gap current in the trigatron. The current jump resulting from bridging of the gap by the streamer(s) is not as obvious in this figure as in Fig. 5 because of the reduced sensitivity, and is marked with an arrow. At this point, occurring about 5 ns after streamer initiation, the switch is still effectively open, and closure must await the formation of an arc. Although the arc formation time is not well defined, it clearly exceeds the streame transit time. To investigate the generality of this conclusion, we measured the arc formation time (defined as the time required for the gap current to reach $95 \%$ of the fnal value, measured from the time when the streamer con-

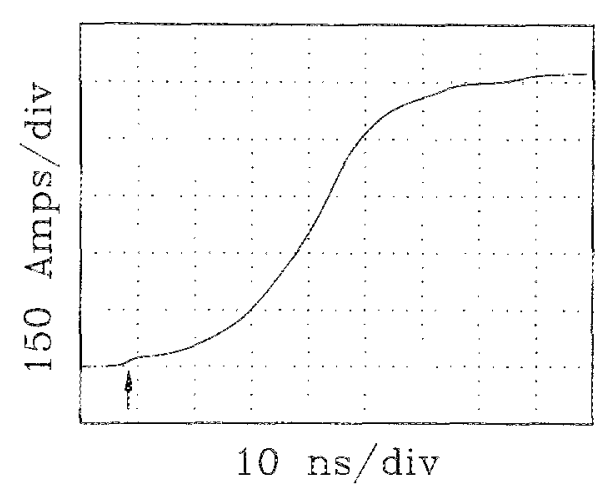

FIG. 8. Oscilloscope rrace of breakdown current obtained with diagnostic 33 showing the complete breakdown process from initiation to gap closure. Conditions were the same as in Fig. 3. The current jump associated with streamer contact is marked with the arrow. 
tacted the distant main gap electrode) for a number of charging voltages, three different trigger pins, and both charging polarities. Figure 9 shows bar graphs of the streamer transit and are formation times for both heteropolar polarity configurations plotted versus total voltage $\left(\left|V_{g}-V_{t}\right|\right)$. Figure 10 shows the arc formation time plotted versus the total voltage for three different trigger pin geometries and for both heteropolar polarity configurations. At the lower charging voltages there was considerable shot-to-shot variation in the arc formation time. The data shown in these figures represent typical values of these times, subjectively averaged over about ten shots per point.

Besides demonstrating the dominance of the arc formation time, these graphs show other interesting features. As expected, the formation time increases with decreasing total voitage. The dependence on voltage is weak for the higher voltages, but becomes much stronger at the lower voltages. The weak dependence of arc formation time on voltage in the likely operating regime is a desirable characteristic since it makes for a low switch-to-switch spread in the closing time for multiple switch systems, and it minimizes drift in the closing time of a single switch caused by small changes in applied voltage or wear of the gap electrodes. For charging voltage near $V_{\mathrm{SB}}$, the formation time was about the same for both polarity configurations, and all pin types. The time increased much faster with decreasing voltage, however, for the $(-+)$ configuration than for the $(+-)$. For the $(+-)$ configuration, the arc formation time is nearly independent of trigger pin geometry for all voltages, whereas
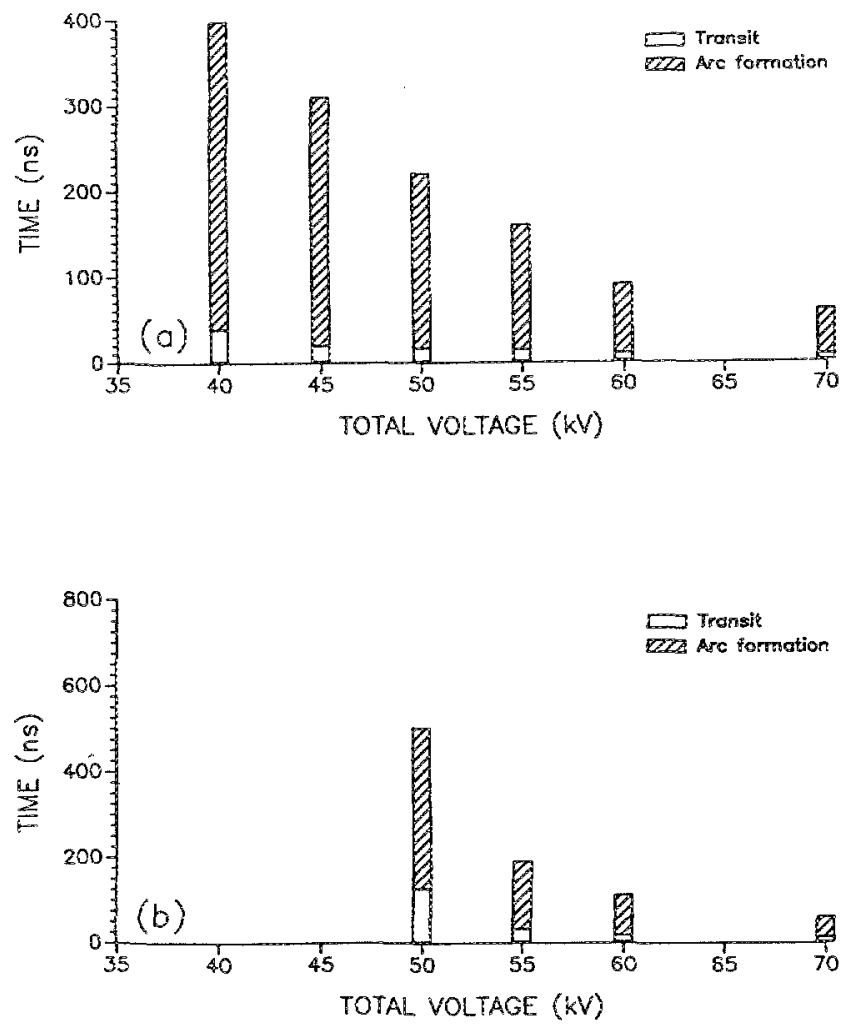

FIG. 9. Comparison of the transit time of the streamers with the arc formation time after streamer contact. Results are shown for both (a) positive trigger, negative main gap $(+-)$ and (b) negative trigger, positive main gap $(-+)$ polarities. Conditions were otherwise the same as in Fig. 3.

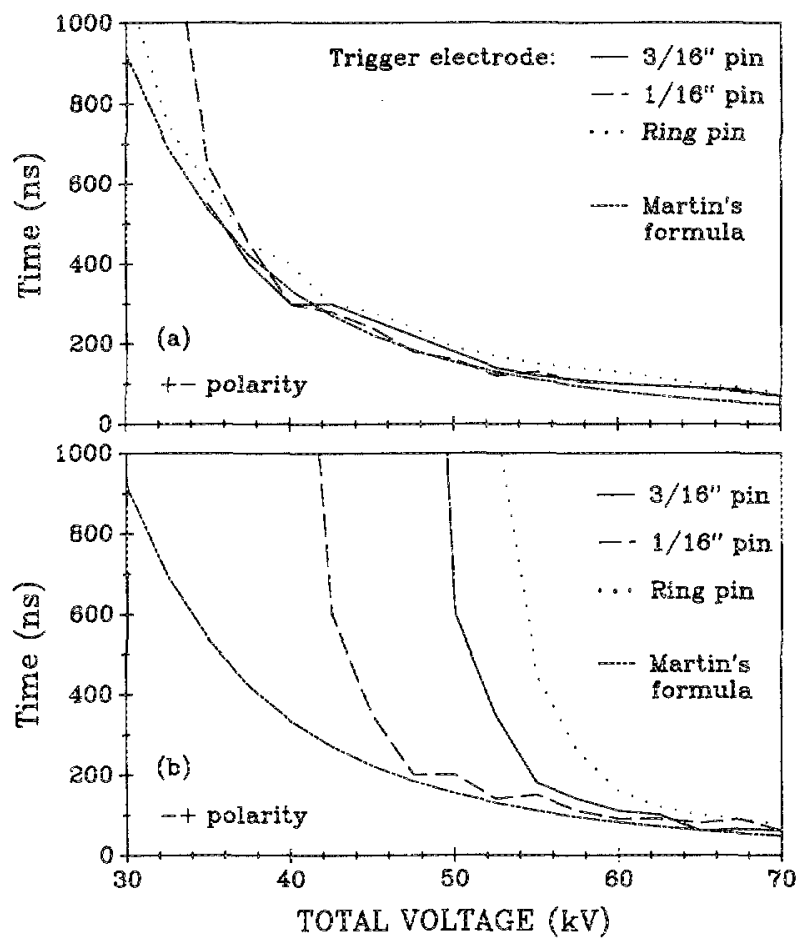

FIG. 10. Plot of arc formation times for varying trigatron gap conditions. The arc formation time was defined to be the time required after streamer contact for the gap current to rise to $>95 \%$ of the steady-state value. Results are shown for several types of trigger electrodes, as well as both (a) positive trigger, negative main gap $(+-)$ and (b) negative trigger, positive main gap $(-+)$ polarities. Gap conditions were otherwise the same as in Fig. 3. Also shown is the prediction of Martin's empirical formula.

there is a strong dependerce with the $(-+)$ configuration for all voltages below about $60 \mathrm{kV}$.

Martin ${ }^{17}$ has developed a phenomenological model for breakdown based on an earlier phenomenological model by Martin. ${ }^{30}$ Among other things, the model predicts the arc formation time for breakdown of gases at pressures around and above atmospheric. Applied to our gap, this formula is ${ }^{18}$

$$
t=k \rho^{2.5}\left(d /\left|V_{g}-V_{t}\right|\right)^{3.5}
$$

where $\rho$ is the mass density of the fill gas, $t$ the arc formation time, $d$ is the gap separation, and $k$ is a constant. For $\rho$ in $\mathrm{g} / \mathrm{cm}^{3}, V_{g}$ and $V_{t}$ in kilovolts, $d$ in centimeters, and $t$ in seconds, Wells gives the constant $k$ as $k=1.1 \times 10^{5} .{ }^{18}$ The times predicted by this formula for our spark gap are also shown in Fig. 10. The formula generally works well for all charging voltages in the $(+-)$ polarity configuration, and for charging voltage near $V_{\mathrm{SB}}$ for the $(-+)$ configuration. It does not, however, predict either the polarity differences we observe at lower voltages, or the variation with differing trigger pins. For the $(-+)$ polarity, this disagreement is outside the range of experimental error.

An important characteristic of spark gap switches is the jitter in gap closing time. Figure 11 shows the closing time jitter measured in our gap plotted versus the total voltage for the same conditions as in Fig. 10. The jitter was measured by recording a number $(\approx 10)$ of main gap current traces on a storage oscilloscope, and noting the range of times recorded. The figure given is the extreme range of times seen, without weighting for the probability of occurrence. The jitter is 


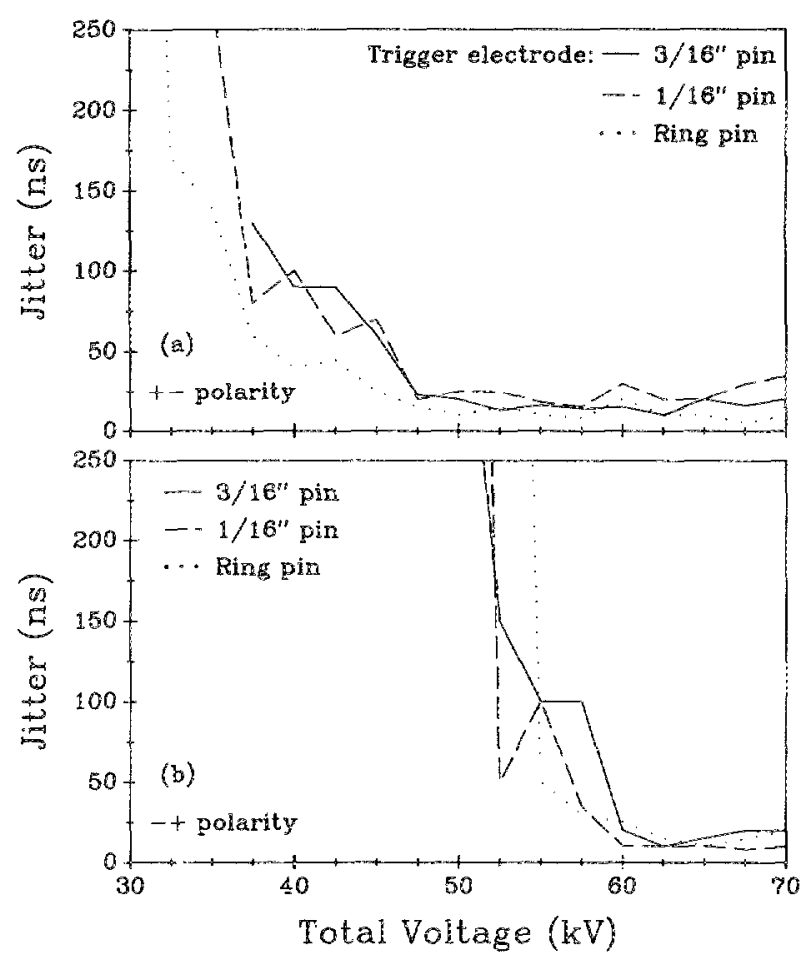

FIG. 11. Plot of jitter in the total gap closing time for varying trigatron gap conditions. Results are shown for several types of trigger electrode, as well as both (a) positive trigger, negative main gap $(+-)$ and (b) negative trigger, positive main gap $(-+)$ polarities. Gap conditions were otherwise the same as in Fig. 3.

roughly independent of total voltage down to a point, and then increases dramatically. To investigate further the processes responsible for this behavior, we measured independertly the jitter in the time of main gap streamer contact with the distant electrode and the overall jitter ir closing time. The results for the configuration producing cathodedirected streamers $(+-)$ are presented in Fig. 12. The jitter in streamer contact time is about $10 \mathrm{~ns}$, independent of the total voltage. The jitter in closing time, on the other hand, is about the same as the jitter in contact time for voltages above about $50 \mathrm{kV}$, but rises sharply for voltages below this value. For the opposite polarity configuration, we have data on the jitter in the time of streamer contact only for total voltage above $60 \mathrm{kV}$. Over this range the behavior was similar to that with the opposite polarity.

These data imply two distinct mechanisms contributing to jitter in closing delay in our gap. One mechanism is associated with jitter in the time of streamer contact with the distant main gap electrode, and the other with jitter in the heating time of the streamer charnel to form the arc. Streak photographs show clearly that in our gap the jitter in the time of streamer contact is aimost entirely due to jitter in the time of streamer formation, rather than jitter in the streamer transit time. As discussed in Sec. IV, we believe the large jitter in streamer formation time is due to the slow rise time of the trigger pulse at the trigger pin tip in our gap, and probably is not intrinsic to the trigatron triggering mechanism.

A large number of distinct physical processes contribute to the arc formation time, and an accurate calculation of this

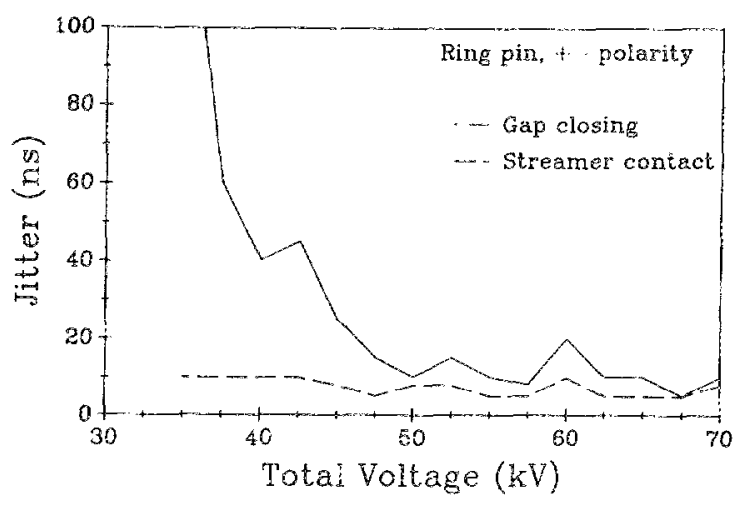

FIG. 12. Comparison of gap closing time jitter and streamer contact jitter for the 6.35-mm-diam ring pin trigger electrode flush with the main electrode, positive trigger, negative main gap $(+-)$ polarity, $F_{1}=10 \mathrm{kV}, \mathrm{N}_{2}$ till at 700 Tor:.

time wonld require a complicated and difficult numerical calculation. We can, however, gain insight into the processes occurring by making simple estimates based on simplifying assumptions expected to either over- or underestimate the formation time. Starting when the initial streamer bridges the gap, power is delivered to the streamer channel at a rate determined by the main gap voltage and the channel current. This power is distributed between excitation of eiectronic, vibrational, rotational, and translational modes of the molecules in the channel in a manner that would require a diffcult and detailed calculation to describe properly. As a limiting case, we can assume that all the electrical power goes into ionizing neutral fill gas molecules. This assumption will certainly predict a lower limit for the arc formation time because a sabstantial portion of the input energy must go into the excitation of other modes of the molecules. Applying this model to the experiment described in Fig. 8, we predict a formation time of the order of $1 \mathrm{~ns}$, abont a factor of 50 faster than observed. If we assume that only a constant fraction, say $f$, of the eiectrical power goes into ionization and adjust $f$ for the best fit with Fig. 8 , we find that the model curve rises too rapidly at first, and too slowly at the end, consistent with heating occurring through a cascade process.

Initially the streamer leaves the channel in a condition in which most gas molecules are in low-lying states, and the ionization process should be described roughly by the Townsend ionization coefficient, $d v / d t=a W_{e} n, W_{e}$ being the electron drift velocity. As a second, complementary, limiting case to that discussed above, we might assume that this relationship would hold over the entire course of the breakdown process, with $\alpha$ and $W_{e}$ being determined by the instantaneous $E$ field and being given by the standard, unperturbed, values. Doing so would give a very long arc formation time because of the collapse of the gap voltage as the are forms and the strong dependence of $\alpha$ on $E$. The much shorter formation times actually observed must be the result of nonzero excited-state densities in the nascent arc channel, or of the appearance of other physical processes such as domain formation. These factors should be much less important during the initial current growth phase. Using appropriate values of $\alpha$ and $\mu_{e}$, we estimate an initial exponential growth time (increase by a factor of $e$ ) for conditions 
in Fig. 8 of about $40 \mathrm{~ns}$, similar to but slower than the $\approx 15 \mathrm{~ns}$ observed. This observation suggests that excited-state densities in the streamer channel are high enough that cascade ionization processes play an important role in ionization production.

A source of the changes in arc formation time with charging voltage may be the changes in the ionization density left behind by the initiating streamer. There is evidence that the ionization density in the streamer does decrease with decreasing applied voltage. We have tabulated the size of the main gap current jump associated with the streamer contacting the distant main gap electrode for several shots at several charging voltages. Although there was considerable shot-toshot scatter, there was a clear trend toward smaller current jump with lower voltage.

\section{Low-voltage operation}

The shutter photos presented in Fig. 3 were of streamers taken with $V_{g}$ close to $V_{\mathrm{SE}}$. The situation changes dramatically with lower charging voltages. Figure 13 shows similar photos taken with $V_{g} \approx 0.5 V_{\mathrm{SB}}$, and the $(+-)$ polarity configuration (producing cathode-directed streamers). The photos in Figs. 13(a)-13 (c) show the initial streamer phase, and Figs. 13(d)-13(f) show the later heating phase. The streamers propagate much slower at this voltage so that the shutter camera has sufficient temporal resolution to catch the streamers in mid-gap and resolve the luminous tip. More important is the erratic, highly forked nature of the streamers seen in these photos. In order to propagate, the streamer must apparently reiy more and more on the space-charge field it induces at its tip as the applied field is decreased. This field is subject to statistical fluctuations which could be responsible for the erratic behavior. A complicating factor that probably also contributes to the different behavior is that because of the slower propagation times, the trigger gap

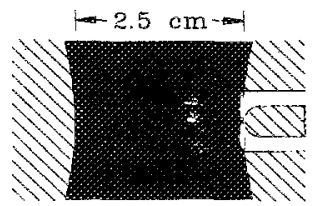

(a) $\mathrm{t}=10 \mathrm{~ns}$

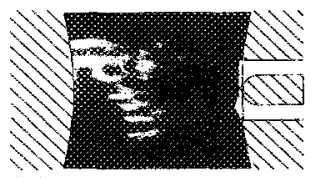

(c) $\mathrm{t}=30 \mathrm{~ns}$

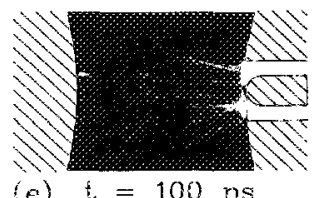

(e) $t=100 \mathrm{~ns}$

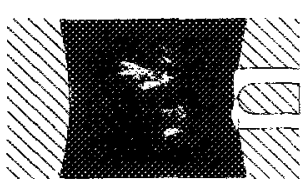

(b) $t=20 \mathrm{~ns}$

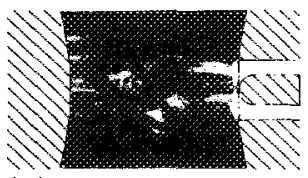

(d)

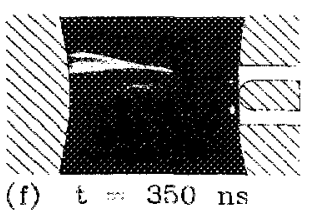

FIG. 13. Sequence of shutter photographs showing the time development of the streamer and arc discharge chanmels for $50 \% W_{\mathrm{SB}}$ main gap charging with positive trigger, negative main gap $(+\cdots)$ polarity. The times indicate the approximate delay since streamers were first initiated. Voltages were $V_{t}=10 \mathrm{kV}, V_{\mathrm{g}}=-30 \mathrm{kV}$. Conditions were otherwise the same as in Fig. 3. breaks down before the streamers have crossed the gap. The emission from the developing trigger spark can be seen at the right-hand edge of the photos.

For these low charging voltages, the photos in Figs. $13(d)-13(f)$ show that the behavior during the heating phase is quite complex. The last photo was taken about $25 \mathrm{~ns}$ before the abrupt rise in current signaling switch closure. In order to protect the camera from the intense emission from the arc a short ( $24 \mathrm{ns)}$ ) charging cable was used for these photos. Figures $13(d)-13$ (f) show that during the heating phase multiple luminous filaments are present, each with what appear to be waves of luminosity propagating along them. In Fig. 13(d), taken just after the first streamers contacted the distant main gap electrode, the localized luminous regions at about mid-gap are probably other streamers still in transit. As time progresses a complex pattern of thin luminous filaments appears. The luminosity of these filaments is nonuniform, and it appears that the filaments become attached to the edge of the hole in the grounded main gap electrode in preference to the trigger pin. We were unable to obtain more detailed information about the motion of these flaments or the regions of luminosity, however, because we could not obtain a sequence of photographs from a single shot.

The situation is quite different with the opposite polarity configuration (producing anode-directed streamers). Figure 14 shows a typical shutter photograph of a streamer in transit under these conditions. The luminosity associated with the tip of the anode-directed streamer is wider and more diffuse than for the cathode-directed streamer, and the propagation velocity (as measured with streak photography) is even slower. The reason for the difference in behavior is probably related to the fact that the anode-directed streamer can decay into a drifting cloud of electrons, whereas the cathode-directed streamer cannot.

The behavior during the heating stage was also quite different in this polarity configuration. Probably because the initial streamer left behind a diffuse ionization distribution, the heating process was much more uniform. We found that
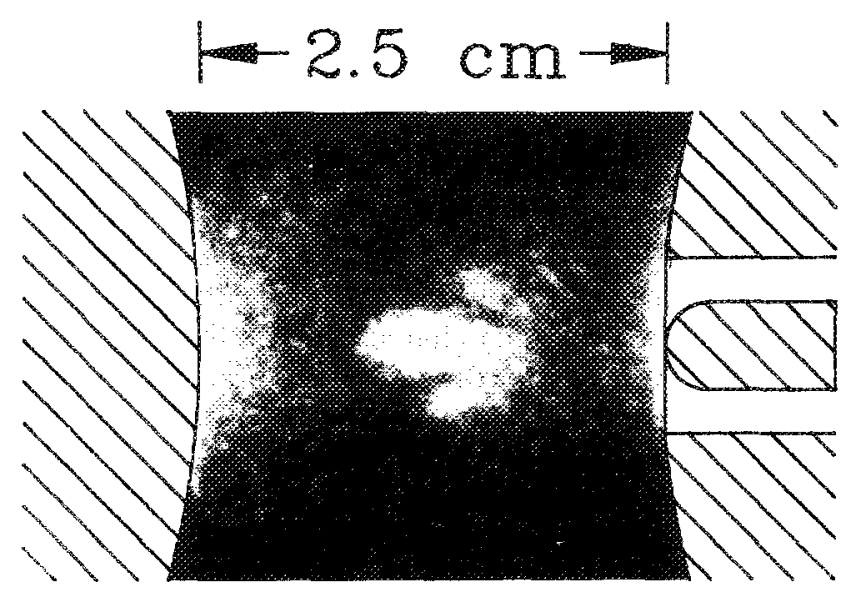

FIG. 14. Shutter photograph of the development of an anode-directed streamer in the negative trigger, positive main gap $(-+)$ polarity for $66 \% V_{\mathrm{SB}}$ main gap charging voltage. Voltages were $V_{t}=-10 \mathrm{kV}, V_{\mathrm{g}}$ $=40 \mathrm{kV}$, and conditions were otherwise the same as in Fig. 3 . 
the luminosity remained diffuse until the late stages of arc formation when one or two filaments would form.

\section{IY. IMPLICATIONS FOR TRICATRON DESIGX}

The experimental results we have obtained show that triggered breakdown of our trigatron spark gap occurs through the sequence of events discussed in Sec. IIl. Physically, the breakdown is a two-step process. First, one or more streamers form and propagate across the main gap. Second, the resulting tonization density, driven by the applied field, increases until the are channel forms and the switch is closed. Closure of the trigger gap is eventually required to complete the circuit, but the are in the gap does not initiate the main gap breakdown process. Instead, the triggering voltage puise initiates breakdown by launching streamers from the enhanced field at the trigger pin tip. Once the streamers have bridged some fraction of the gap, the presence of voltage on the pin may aid the breakdown process, but it is ro longer needed for breakdown to occur.

These conchusions have important implications for the design of trigatrons, and the cuestion of the generality of our observations arises. We nave performed similar experiments for $\mathrm{N}_{2}$ between 250 and 900 Torr; synthetic air and $\mathrm{H}_{2}$ fills at 700 Torr; trigger pin diameters between 0.08 and $0.5 \mathrm{~cm}$; rounded, squared-ofi, and ring-shaped pin tips; pins fush with and recessed below the host electrode surface; charging voltages between about $25 \%$ and $99 \%$ of static self-break voltage (15-62 kV for 700 -Torr $\mathrm{N}_{2}$ ); irigger pulse voltages between 5 and $25 \mathrm{kV}$; and both heteropolar charging configurations ( + , trigger; - , main gap; and vice versa). We have also studied triggering in a smaller $(20 \mathrm{kV})$ trigatron cell. Except for very low charging voltages or very short trigger gaps, breakdown was always initiated by a streamer launched from the trigger pin before breakdown of the trigger gap.

A principal achievement of this research is the clear identification of the streamer mechanism as being responsible for triggering these gaps under normal operating conditions. These streamers are initiated in the high-field surrounding the trigger pin tip, and are not the result of the arc that forms between the trigger pin and the adjacent main gap electrode (the trigger gap). This finding is contrary to the conciusions reported by several authors, and has important implications for trigatron design. In particular, since the trigger vollage collapses upon breakdown of the trigger gap, it is important that one or more streamers be initiated and propagate at least most of the way across the main gap before the enhanced field around the trigger pin tip collapses due to breakdown of the trigger gap. In contrast, if the trigger arc were responsible for initiating breakdown, rapid trigger gap breakdown would be an important design goal. The deleterious effects of too short a trigger gap or too high a trigger voltage have been reported by several authors. $8,9,12-14,16-18,31$

Contrary to another commonly held belief, we found in all cases we studied that the streamer formation and transit time requires only a small fraction of the total breakdown time. Martin ${ }^{17}$ and Wells ${ }^{18}$ have reached a similar conclusion. In our gap we found that the streamer stage was responsible for only $10 \%-20 \%$ of the total breakdown time. Thus efforts at reducing closure delay of trigatron switches should be aimed at accelerating the arc formation time.

Figure 10 shows that Martin's formula [Eq. (1)] for are formation time works remarkably well for the $(+-)$ polarity configuration in our gap. There is significant deviation at the lowest total voltages in that the delay increases more rapidly than the formula predicts. One likely cause of this disagreement is the breakdown of the trigger gap. From shutter photos we know that for total voltages below about $40 \mathrm{kV}$ the trigger gap breaks down before the streamers have traversed the main gap. Since the 50- $\Omega$ trigger generator discharges into a matched $50-\Omega$ load, the total voltage is reduced by hall the trigger voltage ( $5 \mathrm{kV}$ for the data in Fig. 10 ) when the trigger gap closes. For the lowest total voltage for which we have data ( $34 \mathrm{kV}$ ), reducing $V_{t}$ by $5 \mathrm{kV}$ increases the predicted time by a factor of about 1.7 , bringing the model into agreement with experiment for the $(+-)$ polarity.

The disagreement between the model and experiment is more pronounced in the $(-+)$ configuration, and cannot be corrected by including effects due to the early closure of the trigger gap as discussed above. An interesting feature of Eq. (1) is that it has no direct dependence on initiating conditions for the are formation. The equation cannot, therefore, be generally applicable, since it predicts arc formation in a set time, independent of the plasma density of the initiating column, whereas in the limiting cases in which this density is either very low or very high, this time must change. We speculate that the reason for the strong disagreement at lower charging voltages in Fig. 10 for the $(-+)$ polarity is that the plasma density left behind by the initiating streamer drops suficiently to impact the formation time. As discussed in the previous section, we find that for the $(+-)$ configuration the streamers remain filamentary down to the lowest voltages at which the gap will trigger, and that the electron density in the streamer column does not depend strongly on the charging voltage. For the $(-+)$ configuration, on the other hand, the streamers become diffuse as the voltage is decreased below $V_{\mathrm{SB}}$, and the plasma density left by the streamer decreases strongly with charging voltage. We would then expect that Eq. (1) would remain applicable over a larger total voltage range for the $(+-)$ configuration than for the $(-+)$, and this is, in fact, what we observe.

Fitter in the closing delay time limits the applicability of trigatrons. In our gap we found that for charging voltages near $H_{S B}$ the main source of jitter was shot-to-shot variation in the formation time of the streamer. For the $(+-)$ polarity configuration (the one most commonly used), this jitter remained roughly $10 \mathrm{~ns}$, independent of charging voltage, whereas the jitter in arc formation time increased rapidly below abost $70 \%$ of $V_{\mathrm{SB}}$. For the opposite $(-+)$ polarity configuration, we have data on the jitter in streamer contact time only for charging voltage above $82 \% V_{\mathrm{SB}}$. Over this range, the behavior was similar to the $(t-)$ confguration. We believe the primary cause of the jitter in streamer formation time in our gap was the slow rise time of the voltage pulse at the trigger pin tip rather than some basic physical mechanism. Although the trigger generator had a fast 
rise time $(\approx 4 \mathrm{~ns})$, the trigger pin was connected as shown in Fig. 2 to the coaxial cable from the generator through $a \approx 50$ $\mathrm{cm}$ length of rod in a coaxial, but definitely nonconstantimpedance, geometry, and this connection slowed the rise kime at the trigger pin tip substantially. Accurate measurement of the voltage at the trigger pin tip is dificult, but several indirect measurements place the rise time at the tip in the range of $10-20 \mathrm{~ns}$. With the high-field enhancement rear the trigger pin tip, streamers can form in the highest-field regions relatively early in the voltage pulse. The volume of the regions capable of initiating a streamer then increases as the voltage rises. A faster rising trigger voltage pulse would be expected to synchronize streamer formation better in these volumes, thereby reducing jitter.

An attractive feature of the trigatron spark gap in some applications such as crowbaring and high repetition rate systems is the ability of the gap to trigger with a low charging voltage. Triggering with $25 \%$ of $V_{\mathrm{SE}}$ is commonly reported. Unfortunately, delay and jitter increase strongly with charging voltage below about $70 \%$ of $V_{\mathrm{SB}}$. Since both the delay and the jitter arise mainly from the arc formation stage of breakdown under these conditions, further study aimed at improving trigatron operation in this regime should be concentrated on this stage. The photos in Figs. 13 and 14 clearly show that the processes involved are complicated. It is remarkable that a simple phenomenological formula such as Eq. (1) is able to predict closing times in such a regime.

Since cathode-directed streamers are observed to produce a much more filamentary ionization density at these low charging voltages than do anode-directed streamers, the observation that the arc formation time is shorter for the polarity configuration $(+-)$ producing cathode-directed streamers suggests that the conditions left by the streamer phase of breakdown can affect the arc formation phase. Thus, it might be possible to decrease the delay time for low charging voltages by clever design that encourages single filament formation early in the arc formation process. Wells found a correlation between jitter and number of initial streamer channels, and suggested the jitter might be minimized by designing the trigatron so that only a single streamer forms. ${ }^{18}$ Much more work is required, however, before specific design improvements can be suggested.

Erosion of the trigger pin tip is a problem in some high current trigatrons. Typically the circuit is closed by forming an arc from the distant main gap electrode to the trigger pin, and a second are from the trigger to the adjacent main gap electrode. The shape and surface condition of the trigger pin plays a role in determining the triggering characteristics of the switch. High current arcs forming to the pin may erode it, changing these characteristics with operating time. Through careful design, it may be possible to cause the main arc to form directly between the main gap electrodes, without using the trigger pin as an intermediary. Doing so would be expected to increase the lifetime and reliability of high current trigatrons. For experiments conducted using a ringshaped trigger electrode, the main gap arc appeared to be rooted either on the main gap electrode or on a point on the surface insulator close to it. This observation provides at least some encouragement for such attempts.

\section{SUMMARY}

We have presented results from an empirical study of triggering in trigatrons that clearly shows that breakdown is initiated by streamers in these devices under a wide range of conditions. These streamers form in the enhanced field region surrounding the trigger pin tip, anc are not the result of the are which forms later between the trigger pin and the adjacent main gap electrode. Switch closure was shown to be a two-step process, involving first streamer formation and propagation across the main gap, followed by a heating process that converts the resistive streamer channel into a highly conductive arc. The details of this heating process are not well known, but for charging voltages near $V_{\mathrm{SB}}$, they appear to be relatively straightforward. For lower charging voltages, however, the heating process appears to become much more complex.

\section{ACKNOWLEDGMENTS}

F. E. Peterkin was supported by a National Science Foundation Fellowship. We acknowledge helpful discussions with G. Schaefer, T. Martin, R. Gripshover, E. Ball, S. Moran, R. DeWitt, and E. Kunharat.

'J. D. Craggs, M. E. Haine, and J. M. Meek, J. Inst. Electr. Eng. 93A, 963 (1946).

2T. E. Broadbent, Br. J. Appl. Phys. 8, 37 (1957); T. E. Broadbent and A. H. A. Shlash, Br. J. Appl. Phys. 14, 687 (1963).

${ }^{3} \mathrm{~W} . \mathrm{H}$. Lupton, in Proceedings of the 5th International Conference on Ionization Phenomena in Gases, Munich, 1961 (North-Holland, Amsterdam, $1961)$ p. 2059.

${ }^{4}$ J. R. Wilkinson, J. Inst. Electr. Eng. 93A, 1090 (1946).

${ }^{5}$ A. M. Sletten and T. J. Lewis, Proc. Inst. Electr. Eng. 104C, 54 (1957). 'W. Lampe, Elektrotech. Z. A 83, 591 (1962).

"R. F. Saxe, in Proceedings of the 5th International Conference on Ionization Phenomena in Gases, Munich, 1961 (North-Holland, Amsterdam, $1961)$, p. 1033.

${ }^{8}$ P. I. Shkuropat, Zh. Tekh. Fiz. 30, 954 (1960) [Sov. Phys. Tech. Phys. 5 , 895 (1961)].

9P. I. Shkuropat, Zh. Tekh. Fiz. 39, 1256 (1970) [Sov. Phys. Tech. Phys. $14,943(1970)]$

${ }^{10}$ Y. Yoshida and X. Sugita, Tech. Rep. Tohoku Univ. 39, 365 (1974); Y. Yoshida and K. Sugita, J. Phys. Soc. Jpn. 38, 1229 (1975).

"Y. Yoshida, J. Phys. Soc. Jpn. 42, 1404 (1977).

${ }^{12}$ A. S. El'chaninov, V. G. Emel'yanov, V. M. Koval'chuk, G. A. Mesyats, and Yu. F. Potalitsyn, Prib. Tekh. Eksp., 103 (1974) [Inst. Exp. Tech., 416 (1974)!.

${ }^{13}$ A. S. El'chaninov, V. G. Emel'yanov, B. M. Koval'chuk, G. A. Mesyats, and Yu. F. Potalitsyn, Zh. Tekh. Fiz. 45, 86 (1975) [Sov. Phys. Tech. Phys. 20, 51 (1975)].

${ }^{14}$ V. G. Emel'yanov, B. M. Koval'chuk, V. A. Lavrinovich, G. A. Mesyats, and Yu. F. Potalitsyn, Prib. Tekh. Eksp., 89 (1975) [inst. Exp. Tech., $1[14(1976)]$.

${ }^{15}$ V. V. Kremnev, M. V. Novakovskii, and Yu. F. Potalitsyn, Fiz. Plazmy 11, 1285 (1985) [Sov. J. Plasma Phys. 11, 738 (1985)].

${ }^{16} \mathrm{R}$. E. Wootton in Digest of Technical Papers, Sth IEEE Pulsed Power Conference, edited by P.J. Turchi and M. F. Rose (IEEE, New York, 1985), p. 258.

${ }^{17}$ T. H. Martin in Digest of Technical Papers, 5th IEEE Puised Power Conference, edited by P.J. Turchi and M. F. Rose (IEEE, New York, 1985), p. 74.

${ }^{18}$ J. Wells, MSEE thesis, University of New Mexico, 1987.

${ }^{15}$ M. R. Wages, G. Schaefer, K. H. Schoenbach, and P. F. Williams, in $D_{i-}$ gest of Technical Papers, 5th IEEE Pulsed Power Conference, edited by P. J. Turchi and M. F. Rose (IEEE, New York, 1985), p. 414.

${ }^{20}$ F. E. Peterkin, P. F. Williams, and G. Schaefer, in Digest of Technical Papers, 6th IEEE Pulsed Power Conference, edited by B. H. Bernstein and P. J. Turchi (IEEE, New York, 1987), p. 534. 
${ }^{21}$ F. E. Peterkin and P. F. Williams, Appl. Phys. Lett. 53, 182 (1988).

${ }^{22}$ T. Y. Chang, Rev. Sci. Instrum. 44, 405 (1973).

${ }^{23}$ H. Domingos, Puise Power Effects in Discrete Resistors, AFWL Report No. AFWL-TR-76-120 (1976).

${ }^{24} \mathrm{~F}$. Llewellyn-Jones, in Electrical Breakeiown and Discharges in Gases: Fundamental Processes and Breakdown, edited by E. E. Kunhardt and L. H. Leussen (Plenum, New York, 1983), p. 50.

${ }^{25}$ C. Grey Morgan, in Electrical Breakdown of Gases, edited by J. M. Meek and J. D. Craggs (IEEE, New York, 1985), p. 414.
26. W. Dotchin and E. L. Chupp J. Chem. Phys. 59, 3960 (\$973).

${ }^{27}$ S. X. Dhali and P. F. Williams, Phys. Rey. A 31,1219 (1985); S. K. Dhali and P. F. Williams, J. Appi. Phys. 62, 4696 (1987).

${ }^{28} \mathrm{C}$. Wu and E. E. Kunhardt, Phys. Rev. A 37, 687 (1988).

${ }^{29}$ R. S. Sigmond, J. Appl. Phys. 56, 1355 (1984).

${ }^{30}$ J. C. Martin, A WRE Report No. \$SWA/JCM/704/49 (1970).

${ }^{31}$ A. I. Gerasimov, G. D. Kuleshov, A. I. Pavlovski, S. Ya. Slysarenko, and A. S. Fedotkin, Briboty Tekn. Eksp. 18, 111 (1975) [Inst. Exp. Tech. 18, 1455 (1975)]. 